\title{
Aspectos morfométricos de Haemonchus contortus (Rudolphi, 1803) procedentes de caprinos (Capra hircus) da região semiárida do Rio Grande do Norte, Brasil
}

\author{
Morphometric aspects of Haemonchus contortus (Rudolphi, 1803) from \\ goats (Capra hircus) of semiarid region of Rio Grande do Norte, Brazil \\ Janilene de Oliveira Nascimento ${ }^{*}$, Josivania Soares Pereira², Zuliete Aliona Araújo de Souza Fonseca², \\ Wesley Adson Costa Coelho ${ }^{2}$, Ericka Natália Bessa², Sílvia Maria Mendes Ahid ${ }^{2}$
}

\begin{abstract}
RESUMO: No nordeste brasileiro, Haemonchus contortus é considerado um nematódeo gastrintestinal de grande relevância médico-veterinária, por causar queda de produção, atraso no desenvolvimento, perdas econômicas e mortes de pequenos ruminantes. O objetivo do presente trabalho foi identificar as variaçôes morfométricas e os tipos de apêndices vulvares existentes em $H$. contortus provenientes de caprinos da região semiárida do Rio Grande do Norte. Utilizaram-se 100 exemplares de $H$. contortus, oriundos do acervo do Laboratório de Parasitologia Animal da Universidade Federal Rural do Semiárido (UFERSA), onde eles foram montados entre lâmina e lamínula com glicerina, para mensuração em ocular micrométrica em objetiva de 10X, com fator de correçáo de 1,3636. O comprimento corporal dos machos foi inferior ao das fêmeas $(\mathrm{p}<0,05)$, os quais apresentaram respectivamente médias \pm desvio padrão de 13,26 \pm 1,15 e 18,32 $\pm 1,39 \mu \mathrm{m}$. Quanto ao tipo de apêndice vulvar encontrado nas fêmeas, $72 \%$ foram do tipo linguiforme, $18 \%$ do tipo liso e $10 \%$ do tipo botão. Esses dados contribuem de forma significativa para a taxonomia e diferenciação desses endoparasitas, principalmente na regiáo do semiárido do Nordeste, onde a haemonchose tem papel preocupante para a caprinocultura.
\end{abstract}

PALAVRAS-CHAVE: Capra hircus; morfometria; nematódeo.

\begin{abstract}
In northeastern Brazil, Haemonchus contortus is considered a gastrointestinal nematode with great veterinary relevance, causing reduced production, delayed development, economic losses and death of small ruminants. The objective of this study was to identify the morphometric variation and the types of vulvar appendages in $H$. contortus from goats in the semiarid region of Rio Grande do Norte. We used 100 units of $H$. contortus, from the collection of the Laboratory of Animal Parasitology of Federal Rural University of the Semi-Arid (UFERSA), where they were mounted between slide and coverslip with glycerin, to measure in micrometer ocular at $10 \mathrm{X}$ objective with correction factor of 1.3636 . The body length of males was lower than females $(\mathrm{p}<0.05)$, which showed, respectively, mean \pm standard deviation of $13,26 \pm 1,15 \mathrm{e}$ $18,32 \pm 1,39 \mu \mathrm{m}$. Regarding the type of vulvar appendage found in females, $72 \%$ were linguiforme type, $18 \%$ flat type and $10 \%$ button. These data contribute significantly to the taxonomy and differentiation of these endoparasites, especially in northeastern semi-arid region, where haemonchose has a worrisome role for raising goats.
\end{abstract}

KEYWORDS: Capra hircus; morphometry; nematode.

\footnotetext{
'Laboratório de Parasitologia Animal do Departamento de Ciências Animais da Universidade Federal Rural do Semiárido (UFERSA) - Mossoró (RN), Brasil. 2Departamento de Ciências Animais da UFERSA - Mossoró (RN), Brasil.

*Autor correspondente: janilenecat16@hotmail.com

Recebido em: 24/10/2012. Aceito em: 21/10/2013
} 
O Haemonchus contortus é um nematódeo de ruminantes que causa grandes perdas econômicas. No Brasil, especificamente na regiáo semiárida do Nordeste, as maiores perdas desses animais acontecem no final da estação chuvosa e início da seca, quando ocorre um aumento da intensidade de infecçáo por esse parasito. Esse fato estimula o interesse no estudo da morfologia e biologia desse organismo (Costa; VieIra, 1984; Padilha, 1996).

Dentre as diversas pesquisas científicas realizadas para estudo morfológico dos endoparasitos, a morfometria é considerada um importante aspecto para diagnóstico específico, uma vez que permite avaliar a variação intra e interespecífica (Grisı, 1974; VICENTE et al., 1993). Diferenças no tamanho de estruturas internas auxilia o diagnóstico das espécies que acometem os animais domésticos e selvagens, permitindo avaliar infecçôes cruzadas entre espécies (Gibbons, 1979). Diferenças nas medidas e nos caracteres morfológicos vêm sendo citadas como uma possível adaptação às diferentes regióes geográficas, principalmente àquelas de clima com estação seca prolongada (JACQUIET et al., 1997).

$\mathrm{O} H$. contortus tem sido bastante estudado em todo o mundo, tanto morfometricamente como biologicamente (ROBERTS et al., 1954; Mejia-Garcia; Diaz-Benitez, 1987; Lichtenfels et al., 1994). Nessa linha, o presente trabalho objetivou identificar as variaçóes morfométricas e identificar os tipos de apêndices vulvares de $H$. contortus obtidos de infecçôes naturais em caprinos da regiáo semiárida do Rio Grande do Norte.

Foram utilizados exemplares de $H$. contortus recuperados de caprinos leiteiros do município de Afonso Bezerra, Rio Grande do Norte, e pertencentes ao acervo do Laboratório de Parasitologia Animal da Universidade Federal Rural do Semiárido (UFERSA) - Mossoró, Rio Grande do Norte, conservados em soluçáo de formol ácido acético (AFA). Os exemplares foram submetidos à sexagem, selecionando-se 50 machos e 50 fêmeas para o estudo, os quais foram montados entre lâmina e lamínula com glicerina e mensurados em ocular micrométrica com objetiva de 10X, com fator de correçấo de 1,3636 (Fig. 1). Os caracteres analisados nas fêmeas foram: comprimento total do corpo, distância do ânus à extremidade posterior e tipo de

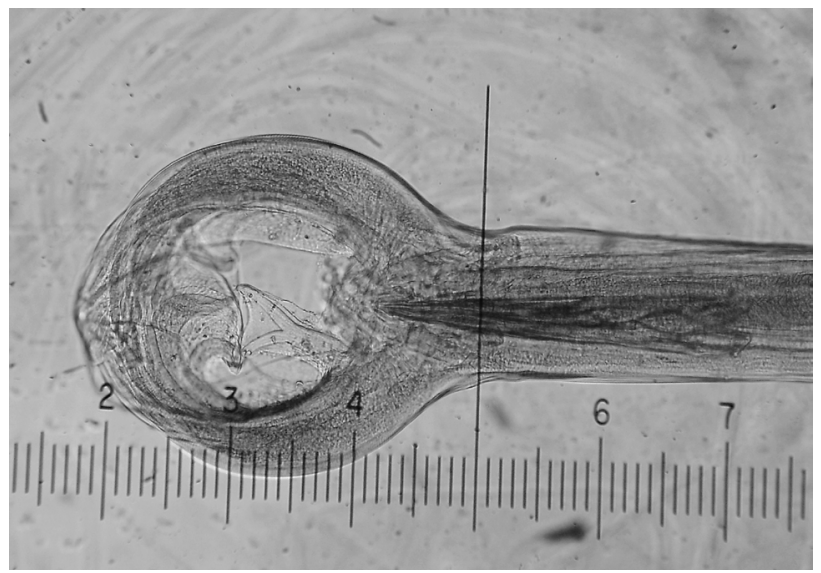

Figura 1. Exemplar macho de Haemonchus contortus, mostrando a região dos espículos com ocular micrométrica em objetiva de 10X. apêndice vulvar. Nos machos, os caracteres foram: comprimento total do corpo e comprimento dos espículos.

Para a análise estatística, os dados foram expressos em média e desvio padrão e avaliados pelo programa estatístico Statistical Package for Social Science (SPSS), versão 17.0 (SPSS. Inc, Chicago, IL, EUA). Após o teste de normalidade de Shapiro-Wilk, aplicou-se o teste $t$ para amostras independentes.

Dos caracteres morfométricos analisados, observou-se que o comprimento corporal médio foi de 13,26 $\pm 1,15 \mathrm{e}$ $18,32 \pm 1,39 \mathrm{~mm}$ nos machos e fêmeas, respectivamente, sendo considerados estatisticamente distintos $(\mathrm{p}<0,001)$. Nos machos, o comprimento dos espículos foi de 385,62 $\pm 24,18 \mu \mathrm{m}$ $\mathrm{e}$, nas fêmeas, a distância do ânus à extremidade posterior foi de $313,35 \pm 60,70 \mu \mathrm{m}$. Estudo realizado por ARAújo; Rodrigues (2002) com exemplares de $H$. contortus provenientes de caprinos na regiáo semiárida da Paraíba demonstrou comprimento médio do corpo de 14 e 19,48 mm para machos e fêmeas, respectivamente, variando de 14 a $27 \mathrm{~mm}$ nas fêmeas estudadas. Já para o comprimento do espículo nos machos, a média encontrada foi de $442 \mu \mathrm{m}$, maior que o encontrado no presente estudo. Segundo Silva; Rodrigues (2003), quando estudados os $H$. contortus provenientes do semiárido paraibano na cidade de Patos e de Sobral no estado do Ceará, o comprimento do corpo foi de 16,00 \pm 0,6 e $15,8 \pm 3,1 \mathrm{~mm}$, respectivamente. Para o comprimento dos espículos, o mesmo estudo obteve $269,5 \pm 30,2 \mathrm{e}$ $348,8 \pm 47,6 \mu \mathrm{m}$, respectivamente.

Quanto ao tipo de apêndice vulvar encontrado nas fêmeas, observou-se que $72 \%$ foram do tipo linguiforme, $18 \%$ do tipo liso e $10 \%$ do tipo botão. Esses valores divergem do encontrado por Mejia-Garcia; Diaz-Benitez (1987) no México e por Araújo; Rodrigues (2002) na cidade de Patos, no estado da Paraíba, que verificaram 52\% do tipo liso, 20\% do tipo linguiforme e $58 \%$ do tipo botão. JACQUIET et al. (1995), por sua vez, encontraram predominância do processo também do tipo linguiforme. Os resultados do presente trabalho foram concordantes ainda com os resultados obtidos por Almeida (1935) e Grisi (1975), na Bahia.

As características morfométricas encontradas contribuem de forma significativa para a taxonomia e diferenciação desses endoparasitas, principalmente na regiáo do semiárido do Nordeste, onde a haemonchose tem papel preocupante para a caprinocultura.

\section{AGRADECIMENTOS}

Ao Conselho Nacional de Desenvolvimento Científico e Tecnológico (CNPq) pela concessáo da bolsa para o desenvolvimento do trabalho e ao Laboratório de Parasitologia Animal da UFERSA. 


\section{REFERÊNCIAS}

ALMEIDA, J.L. Revisão do gênero Haemonchus Coob, 1898 (Nematoda: Trichostrongylidae). Memórias do Instituto Oswaldo Cruz, v.30, p.57-114, 1935.

ARAÚJO, M. M.; RODRIGUES, M. L. A. Estudo morfométrico e variação do apêndice vulvar de Haemonchus contortus (Nematoda: Trichostrongyloidea) de caprinos e ovinos da região semi-árida da Paraíba - Brasil. Revista Brasileira de Parasitologia, v.2, p.65-67, 2002.

COSTA, C.A.F.; VIEIRA, L.S. Controle de nematódeos gastrintestinais de caprinos e ovinos do estado do Ceará. Sobral: EMBRAPA-CNPC, 1984 (Comunicativo Técnico, 13).

GRISI, L. Variações morfológicas dos espículos e dos processos vulvares de Haemonchus contortus (Rudolphi, 1803), de Capra hircuse Ovis aries L. do estado da Bahia (nematoda, Trichostrongylidae). 1974. 55f. Dissertação (Mestrado em Parasitologia) - Faculdade de MedicinaVeterinária, Universidade Federal de Minas Gerais, Belo Horizonte, 1974.

GRISI, L. Incidence of helminths in Capra hircus L., from Bahia State. Revista Brasileira de Biologia, v.35, p.101-107, 1975.

GIBBONS, L.M. Revision of the genus Haemonchus Cobb, 1898 (Nematoda: Trichostrongylidae). Systematic Parasitology, v.1, p.3-24, 1979.

JACQUIET, P.; CABARET, J.; CHEIKH, D.; THIAM, A. Identification of Haemonchus species in domestic ruminants based on morphometrics of spiculis. Parasitology Research, v.83, p.82-86, 1997.
JACQUIET, P.; HUMBERT, J.F.; COMES, A.M.; CABARET, J.; THIAM, A.; CHEIKH, D. Ecological, morphological and genetic characterization of sympatric Haemonchus spp. species in domestic ruminants based on morphometric of spicules. Parasitology Research, v. 1 10, p.483-492, 1995.

LICHTENFELS, J.R.; PILITT, P.A.; HOBERG, E.P. New morphological characters for identifying individual specimens of Haemonchus spp. (Nematoda: Trichostrongyloidea) and a key to species in ruminants of North America. Journal of Parasitology, v.80, p.107-119, 1994.

MEJIA-GARCIA, R.A.; DIAZ-BENITEZ, A. Variación morfológica del apéndice vulvar de Haemonchus contortus y $\mathrm{H}$. similis en bovinos y caprinos. Revista Ibérica Parasitologia, v.47, p.365-375, 1987.

PADILHA,T. Controle dos nematódeos gastrintestinais em ruminantes. Coronel Pacheco: EMBRAPA-CNPL, 1996.

ROBERTS, F.H.S.; TURNER, H.N.; MCKEVETT, M. On the specific distinctness of the ovine and bovine "strains" of Haemonchus contortus (Rudolphi) Cobb (Nematoda: Trichostrongylidae). Australian Journal of Zoology, v.2, p.275-295, 1954.

SILVA, W.W.; RODRIGUES, M.L.A. Variações morfológicas em populações de Haemonchus contortus (Rudolphi, 1083) Cobbold, 1898 (Nematoda: Trichostrongylidae) de caprinos (Capra hircus) no semi-árido nordestino do Brasil. Ciência Animal, v.13, p.107-109, 2003.

VICENTE, J.J.; RODRIGUES, H.O.; GOMES, D.C.; PINTO, R.M. Nematóides do Brasil. Parte V: Nematóides de Mamíferos. Revista Brasileira de Zoologia, v.4, p.1-452, 1993. 